Cuadernos de Ilustración y Romanticismo

Revista Digital del Grupo de Estudios del Siglo XVIII

Universidad de Cádiz / ISSN: 2173-0687

$\mathrm{n}^{\circ} 25$ (2019)

\title{
LA EDICIÓN PERDIDA DE WIELAND EN ESPAÑA (CA. 1818)
}

José Manuel Correoso Rodenas (Universidad de Castilla-La Mancha)

Recibido: 02-01-2019/Revisado: 04-04-2019

Aceptado: 04-04-2019 / Publicado: 20-12-2019

RESUMEN: El presente estudio tiene como objetivo rescatar del olvido una edición española de principios del siglo XIX de una de las obras fundacionales de la literatura estadounidense: Wieland, de Charles Brockden Brown. El hecho de que esta novela llegase al panorama cultural español a través de una traducción francesa previa ha hecho que haya pasado desapercibida hasta el día de hoy. Finalmente, la publicación de Vieland en una España en la que aún actuaba la Inquisición, hace que este trabajo sea especialmente relevante.

Palabras Clave: Luis Monfort, Charles Brockden Brown, Vieland, traducción, literatura gótica.

\section{THE LOST EDITION OF WIELAND IN SPAIN (CA. 1818)}

ABSTRACT: The main objective of this article is to recover an early $19^{\text {th }}$ century Spanish edition of one of the first important novels of the literature produced in the United States: Wieland, by Charles Brockden Brown. One of the reasons why Vieland has been obviated by most of the critics for two centuries is because it was translated from a previous French version. Finally, the publication of this novel in a cultural panorama still influenced by the Spanish Inquisition makes the relevance and importance of this article even higher. KeYwORDs: Luis Monfort, Charles Brockden Brown, Vieland, translation, Gothic Literature. 


\author{
«Leed y estremeceos; nada \\ bay aqui de fabuloso»
}

\title{
INTRODUCCIÓN
}

En I992, la editorial Valdemar, dentro de su colección «Gótica», dedicada al género de terror, publicó una traducción del clásico de Charles Brockden Brown (I77I-18Io) Wieland (I798), 'bajo el título Wieland, o la transformación. La traducción de los originales corrió a cargo de Juan Ignacio de Laiglesia, y el volumen se completaba con una introducción del filósofo Agustín Izquierdo. Más allá de los errores históricos y teóricos que este prólogo incluye (como decir que Brown fue el primer escritor profesional de Estados Unidos o que Wieland pertenece a la vertiente, propia de Ann Radcliffe -I764-I823-, del «explained gothic», por ejemplo), también ofrece un breve párrafo en el que se incluye la fortuna crítica del autor de una forma superficial. Por ejemplo, se muestran testimonios de la fama que Brown había ganado hacia I85o (presumiblemente en Estados Unidos, aunque no se especifica) y las traducciones que de sus obras se habían realizado al francés y al alemán. ${ }^{2}$ Sin embargo, la introducción concluye con la siguiente frase: «La obra de Brown aparece ahora traducida al español por primera vez» (Izquierdo, 1992: 10).

El objetivo de este artículo es demostrar la existencia de una versión previa Wieland, publicada en España en una fecha tan temprana como es I818. Estas traducciones tempranas fueron enmascaradas bajo la apariencia de unas traducciones de novela francesa, como se verá más abajo. En cualquier caso, la investigación bibliográfica llevada a cabo ha demostrado que la novela más famosa de Charles Brockden Brown llegó a las letras españolas, y la importancia que esto tuvo en el contexto cultural de la primera mitad del siglo XIX en España.

\section{Primer paso: Francia}

Según los documentos que se custodian en la Bibliothèque Nationale de France (Reference: FRBNF33388636), el año I808 habría sido testigo del primer «viaje» transatlántico de Charles Brockden Brown y sus obras. Ese año, el escritor francés Pigault-Maubaillarcq (?-1839)3 publicó una traducción de Wieland, titulada La famille Wieland, ou Les prodiges, traduction libre d'un manuscrit américain. Esta versión apócrifa sería el medio por el que Brockden Brown llegaría a España. ${ }^{4}$ Como se puede apreciar,

I Para el presente estudio se utilizará la siguiente edición: Charles Brockden Brown, Wieland and Memoirs of Carwin the Biloquist, Nueva York, Norton, 20II.

2 La fortuna crítica de Brown ha sido ampliamente estudiada por Cathy N. Davidson, quien afirma lo siguiente: «His Ormond (I799) was the first American novel to be published in England. Almost immediately after Brown's death in I8Io, his friends and fellow writers began promoting a complete edition of his novels, and in 1827 that demand was fulfilled to become another American first. At the same time, still one more first was in the works. In I824, John Neal turned Brown's life into a type of the "plight of the American writer," praised abroad, neglected at home» (Davidson, 2004: 337). Françoise Charras también ha estudiado las traducciones de las obras de Brown, centrándose en la vertiente alemana: «En effet, Wieland at Arthur Mervyn ne seront traduits en allemande qu'en r858» (I999: 19I).

3 Hermano del también escritor Charles-Antoine-Guillaume Pigault de l'Épinoy, más conocido como PigaultLebrun (1753-1835), dedicatario del Wieland de Pigault-Maubaillarcq.

4 Así, Charles Brockden Brown se uniría a la nómina de escritores estadounidenses que fueron traducidos en nuestro país a partir de versiones francesas previas, una práctica que duraría hasta las primeras décadas del siglo xx, como explica Margarita Rigal Aragón en relación a Edgar Allan Poe (I809-1849): «The translations in Historias extraordinarias repeatedly demonstrate a reliance on Baudelaire's French translation. For instance, if we contrast Poe's English and Baudelaire's French versions of "Hans Pfaall", it seems that this early Spanish translation followed Baudelaire's version and not Poe's. Neither Baudelaire nor the Spanish translator include the long explanatory note that Poe attached to the tale to explain the story's resemblance to Mr. Locke's "Moon-story"» (20I4: I5). 
Brown no aparece mencionado ni en la portada de la novela ni en el prólogo («Avertissment») que Pigault-Maubaillarcq escribió. ${ }^{5}$ Esto provocó que tanto el público francés como el español ignorasen su autoría durante estos primeros años.

El primer volumen, como se ha mencionado más arriba, se abre con un pequeño prólogo de una página y media en el que Pigault-Maubaillarcq explica la razón de ser de la novela, presentándose como su autor. Este prólogo destaca los aspectos morales de Wieland, diciendo que es una historia compuesta para prevenir futuros crímenes cometidos a causa del fanatismo: «On n'a eu d'autre but, en imprimant cet Ouvrage, que de presenter quelques points importans de la constitution morale de l'homme; et le lecteur instruir décidera s'il faut classer cet ouvrage parmi les productions frivoles, ou parmi celles dont l'utilité promet un succès durable» (I808: s. p.). Los volúmenes primero y segundo siguen la trama ideada por Brockden Brown, mostrando la historia del viejo Wieland en Europa, cómo abandonó el continente y se estableció en América, su misteriosa muerte y cómo Theodore y Clara continuaron con sus vidas de una forma más o menos apacible hasta la aparición de Carwin. También se narra cómo algunos personajes (Clara, Theodore, Pleyel) comienzan a experimentar hechos perturbadores tras la llegada del infausto personaje. Estos dos volúmenes se cierran con el asesinato que Theodore comete sobre su familia, con él en prisión y con Clara tratando de sobreponerse a estos trágicos episodios. Por otro lado, los volúmenes tres y cuatro abandonan el hilo narrativo creado por Brown, creando una historia completamente nueva. El volumen tres está dedicado a las memorias de Carwin, presentándolo como descendiente de Sebastián de Portugal (I5541578) y rey de un oscuro reino africano gobernado por jesuitas; ${ }^{6}$ el volumen cuatro narra la historia de una rebelión ficticia de los nativos americanos contra el dominio colonial británico, todo ello imbuido del espíritu del Romanticismo, y cómo Carwin convence a las diferentes naciones nativas para que lo coronen como su rey. Finalmente, los familiares de Clara y Pleyel encuentran el escondite de Carwin cerca del Lago Erie y acaban con la rebelión matando a su líder. La traducción española objeto de estudio en este artículo sigue, a grandes rasgos, esta estructura. Debido a esto, cuando se discuta la versión española, las cuestiones acerca de qué variaciones sufrió la obra de Brown solo se contestarán en relación a los dos primeros volúmenes. No obstante, se expondrán algunos aspectos bibliográficos y editoriales que conciernen a la obra en su conjunto.

Más allá de la importancia que esta primera versión francesa pudiese tener, debe tenerse en cuenta el contexto en el que fue concebida. La Francia napoleónica, continuadora de las libertades que la Revolución había traído en materia de cultura y prensa, promovía la publicación de obras extranjeras, aunque la religión jugase un papel importante en las mismas, como es el caso de Wieland. Por otro lado, Francia siempre había constituido un mercado provechoso para los autores de novela gótica, especialmente para Ann Radcliffe, reseñada por el marqués de Sade (1740-18r4) en su Les Crimes de l'Amour (I800), o para William Beckford (I760-1844), cuyo Vathek apareció simultáneamente en francés y en inglés en i786. Este clima de permisibilidad y aceptabilidad que

5 Si Pigault-Maubaillarcq omitió a propósito la autoría de Brown, lo hizo con gran éxito, pues el editor incluyó la siguiente nota al comienzo de cada volumen: «Nous sommes chargés par l'Auteur d'annoncer qu'il met la présente édition sous la sauve-garde des lois et de la probité des citoyens; declarant qu'il poursuivra devant les Tribunaux tout contrefacteur ou distributeur d'éditions contrefaites, et assurant à celui qui les lui fera connaître ou à nous, la moitié du dédommagement que la loi lui accorde. Tout exemplaire qui ne sera pas revêtu de notre signature, est désavoué par l'Auteur, et será regardé comme contrefaçon» (I808: s. p.). La inclusión del subtítulo «traduction libre d'un manuscrit américain» debe entenderse, pues, como un recurso literario, y no como un reconocimiento del origen de la novela.

6 Las referencias a este reino africano, según Françoise Charras, podrían haber sido tomadas de la obra de Jeronimo Lobo (1595-1678) Voyage en Abyssinie (1625-1627). 
Pigault-Maubaillarcq encontró para su traducción sería muy distinto al que encontraría Luis Monfort para la suya.

\section{El DESCUBRIMIENTO}

Antes de entrar a discutir la traducción y sus implicaciones, cabe mencionar cómo se llegó al conocimiento de la existencia de esta traducción. La versión de Monfort es conocida por los investigadores españoles (en su gran mayoría especialistas en el Romanticismo) desde su publicación en I8I8. Así, ha sido mencionada, a través de los años, en diversas publicaciones académicas de distinta índole. De entre todos ellos, el testimonio de María José Alonso Seoane es especialmente interesante debido a que afirma que la versión de Monfort disfrutaba de cierto éxito editorial:

Desde el inicio de la colección de i8I8, Cabrerizo había tenido problemas con algunas de las novelas que iban saliendo y que tenían gran aceptación; en concreto, Ricardo y Sofía, o los yerros del amor, La familia de Vieland o los prodigios y Elena y Roberto, o los dos padres, que acabaron siendo prohibidas por la Inquisición en I8I9 (2002: 13).

Los problemas que Alonso Seoane menciona van a resultar muy relevantes cuando se trate de establecer la importancia de Vieland en un contexto de control inquisitorial sobre la cultura. Sin embargo, a pesar de la cantidad de trabajos en los que La familia de Vieland aparece mencionada, ninguno de ellos la relaciona con Charles Brockden Brown. Esto se puede entender como una carencia en el conocimiento de las letras estadounidenses por parte de los autores de estas obras, pues la mayoría de ellos son críticos e historiadores de la literatura española, por lo que su único interés en la traducción de Monfort habría sido con el objeto de incluirlo en las listas bibliográficas que acompañaban sus publicaciones. Durante el desarrollo de una investigación paralela, el título Vieland apareció entre los documentos que se estaban examinando, y las similitudes con el original Wieland se mostraron evidentes. Una visita a la Biblioteca Nacional sirvió para confirmar estas sospechas: la obra que Luis Monfort había traducido no era un original de Pigault-Maubaillarcq, sino de Brockden Brown. Ahí también se pudo establecer la importancia de esta versión para el desarrollo del Romanticismo español, como se explicará más adelante; una importancia que, debido al enmascaramiento de sus orígenes norteamericanos, ha permanecido obviada durante doscientos años hasta el momento presente.

\section{LA FAMILIA DE VIELAND, Ó LOS PRODIGIOS}

Antes de discutir la traducción y sus características, es necesario decir algo acerca de la figura del traductor. Poco se sabe sobre Luis Monfort, ni siquiera las fechas entre las que transcurrió su vida. Casi toda la información disponible hoy en día sobre él ya apareció en la portada de las distintas ediciones de Vieland. Es fácilmente asumible que esta información la proveyó él mismo al editor. Lo que esta portada dice es que Monfort había completado un Doctorado (puesto que se le nombra como Dr.), presumiblemente en Teología. Esta deducción se puede hacer teniendo en cuenta el breve párrafo que aparece bajo su nombre, en el cual se dice que era «Capellán Párroco del $2^{\circ}$. regimiento del Real Cuerpo de Artillería» y «Secretario de la Subdelegación Castrense de Valencia» [véase la Figura I]. Una investigación más extensa en los archivos el Arzobispado Castrense ha probado que Monfort solo pasó un período corto de tiempo en Valencia, puesto que su 
nombre solo aparece en el libro de bautismos del año 1818.7 Se puede deducir que, tras ese año, fue transferido a un destino diferente. Nada más se sabe acerca de este oscuro sacerdote y traductor el cual, paradójica e inconscientemente, llevó a cabo la tarea de introducir la literatura estadounidense en España.

\section{POR EL DR. D. LUIS MONFORT, Capellan Párroco del $2 .^{\circ}$ regimiento del Real Cuerpo de Artilleria,y Secretario de la Subde- legacion Castrense de Valencia.}

Figura r: Información sobre Luis Monfort que aparece en la portada de Vieland

Teniendo en cuenta la información dada en las secciones previas acerca de la oscuridad en la que Vieland yació durante aproximadamente 150 años, es sorprendente la información de la que se dispone acerca de cómo fue recibida por el público durante la primera mitad del siglo xix. Esta traducción se aleja de esas oscuras novelas góticas que solo tuvieron una única edición. Por el contrario, Vieland disfrutó de cuatro ediciones (I8I8, I826, I830 y I839), según Miriam López Santos (2010: 288). ${ }^{8}$ Un estudio previo, de José F. Montesinos (1982), solo menciona las ediciones de 1830 y 1839, las publicadas por Mariano de Cabrerizo (1785-1868). ${ }^{9}$ Para la presente investigación solo ha sido posible consultar las ediciones de los años 1818, I826 y I839; sin embargo, como se explicará más abajo, no hay razón para pensar que la edición de 1830 incluyese cambios o adiciones sustanciales.

Como se ha dicho más arriba en relación a la versión de Pigault-Maubaillarcq, el Vieland español sigue la misma estructura dividida en cuatro volúmenes, con los dos primeros siguiendo la Wieland de Brockden Brown, y siendo el tercero y el cuarto una narración paralela con Carvino [Carwin] como protagonista. Aparte de esta disposición, debe mencionarse que el primer volumen está dividido en doce capítulos, el segundo en once, el tercero aparece todo comprimido en un único capítulo y el cuarto se divide en diez capítulos. Dicho esto, también es interesante apreciar cómo las distintas ediciones españolas introdujeron variaciones en la organización de los volúmenes. Las ediciones de I818 y I826 se organizaron en cuatro tomos, cada uno de ellos dedicado a un volumen, como una pequeña colección; ${ }^{\text {:o }}$ la edición de 1839, por otro lado, fue publicada en dos tomos: el primero de ellos incluía los volúmenes primero y segundo, y el segundo los

7 Me gustaría expresar mi gratitud para con el Archivo General del Ejército por la ayuda prestada durante la búsqueda de información acerca de Luis Monfort.

8 Serían, sucesivamente: (I818), La familia de Vieland, ó los prodigios [trad. Dr. D. Luis Monfort], Valencia, Imprenta de Estévan; (1826), La familia de Vieland, ó los prodigios [trad. Dr. D. Luis Monfort], Valencia, Imprenta de Gimeno; (I830), La familia de Vieland, ó los prodigios [trad. Dr. D. Luis Monfort], Valencia, Imprenta de Cabrerizo; (1839), La familia de Vieland, ó los prodigios [tran. Dr. D. Luis Monfort], Valencia, Imprenta de Cabrerizo.

9 Mariano de Cabrerizo fue uno de los editores más influyentes durante la primera mitad del siglo XIX, en especial debido a su compromiso con el Liberalismo, lo que le llevó a publicar obras que habían sido previamente censuradas por la Inquisición y el gobierno absolutista. Él mismo escribió sus memorias en I854 (Memorias de mis vicisitudes políticas desde 1820 á 1836 , Valencia, Imprenta de don Mariano de Cabrerizo) donde se puede encontrar más información.

Io La copia que se conserva en la Biblioteca Nacional pertenece a la edición de I8I8, y está incompleta, únicamente estando disponible el primer tomo. Sin embargo, la edición de 1826 es, aparentemente, una reimpresión de la de 1818. Esta sí está completa y sirve para una mejor comprensión acerca de cómo se organizó esta primera edición. 
volúmenes tercero y cuarto. Además, esta edición parece ser una reimpresión barata de las anteriores, sin modificaciones aparentes. ${ }^{\text {II }}$ Continuando con las características formales de las distintas ediciones de Vieland, cada volumen aparecía introducido por un frontispicio. Sin embargo, estas estampas no aparecen en la edición de 1826. La que acompaña al primer volumen de la versión de Monfort parece ser una copia de la ilustración que apareció con el primer volumen de la versión de Pigault-Maubaillarcq (manteniendo incluso el pie de imagen, simplemente traducido) como se aprecia en las Figuras 2 y $3^{:{ }^{12}}$

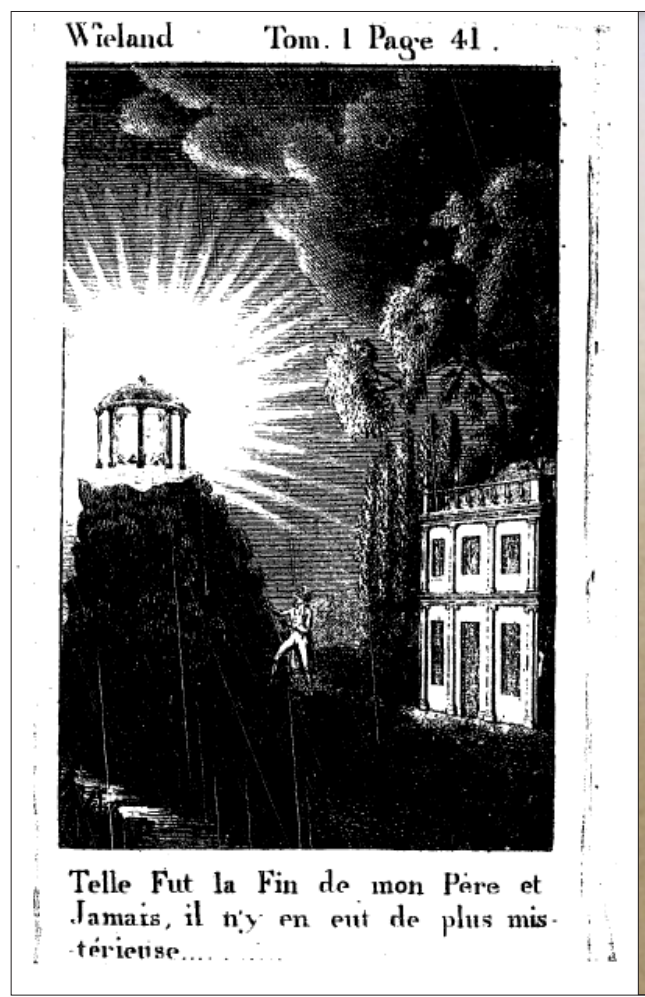

Figura 2: Frontispicio que acompañaba el primer volumen de la versión de Pigault-Maubaillarcq. Fuente: fotografía del autor con permiso de la Bibliothèque Nationale de France.

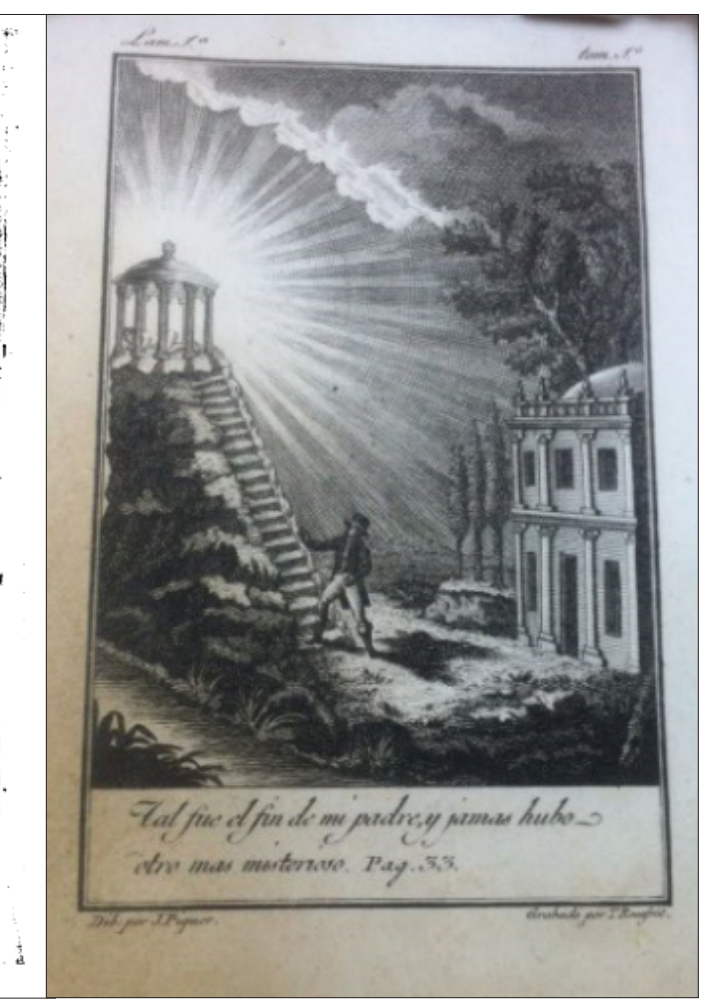

Figura 3: Frontispicio del primer volumen de la edición española de I8I8. Fuente: fotografía del autor con permiso de la Biblioteca Nacional.

Sin embargo, el volumen II incorporaba una nueva imagen que aparecía en la versión francesa, mostrando a Clara en el momento de encontrar los cadáveres de la familia de Teodoro [Theodore] (véase la Figura 4): ${ }^{13}$

Ir Puesto que sólo ha sido posible acceder al tomo IV de la edición de I83o (albergado en la Biblioteca de Cataluña) no se puede afirmar a ciencia cierta cómo se organizaba. Sin embargo, puesto que fue la primera que publicó Mariano de Cabrerizo, la organización pudo haber seguido un modelo similar al de la edición de I839, también publicada por él.

I2 Esta imagen es la única que aparece firmada. Según las firmas conservadas, fue dibujada por un tal J. Piquer y grabada por T. Roafrot. Según E. Benezit, J. Piquer puede referirse a un artista que trabajó en Valencia en el momento en el que el frontispicio fue publicado. Todo lo que se sabe es su nombre, José Piquer II (?-I832), y que fue escultor y grabador cuya obra más famosa es un bajorrelieve realizado para la Real Academia de Bellas Artes de San Carlos de Valencia (I999, vol. XI: I4-I5). No existe información sobre Roafrot.

I3 El autor de esta estampa es desconocido. La única firma reza: «Juln Mas la retocó». 


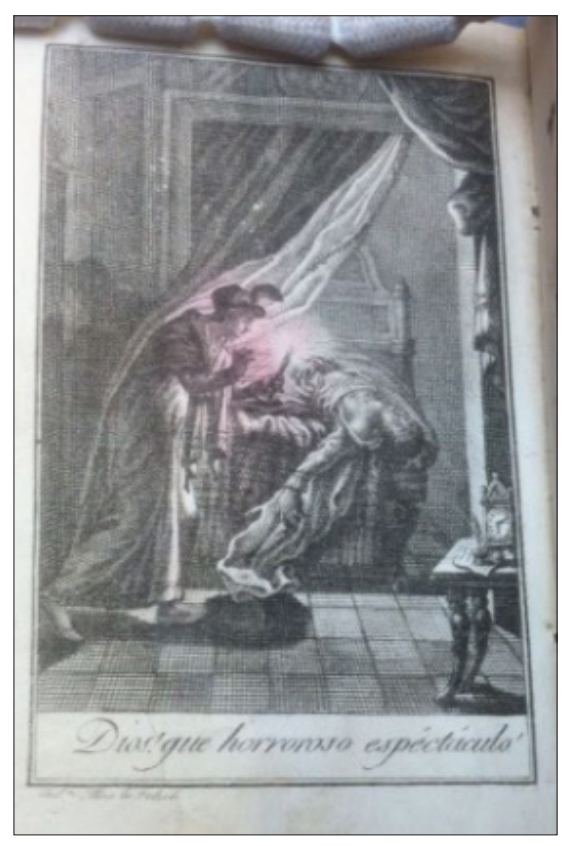

Figura 4: Frontispicio que acompañaba al primer volumen de la edición española de I839. Fuente: fotografía del autor con permiso de la Biblioteca Nacional

Tras la explicación de la apariencia material de Vieland, se procederá al análisis de los textos que tanto el traductor como los editores incluyeron en las distintas ediciones. La edición de i8r8 (impresa y publicada en la Imprenta de Estévan, de Valencia) cuenta con un prólogo sin firma titulado «El traductor», haciendo plausible pensar que fue el propio Monfort quien escribió estas páginas. El prólogo va de la página $\mathrm{V}$ a la XIV, y es principalmente una justificación de cómo la novela puede ayudar a mejorar la moralidad del lector, de modo similar a como Pigault-Maubaillarcq había hecho en su versión una década antes. Por ejemplo, el prólogo español se abre con la frase

Sin la ilustración del Evangelio, y sin el freno dulce y firme con que la Religion dirige las acciones del hombre, ni basta para hacerle feliz toda la virtud que la naturaleza y la sociedad pueden darle, ni menos podrán hacerle verdaderamente virtuoso los mas grandes talentos, ni la mas asombrosa sabiduría (I818: v). ${ }^{14}$

Sin embargo, este no es el único párrafo interesante que Luis Monfort incluyó en su prólogo. En las páginas VII, viII y IX, trata acerca del fanatismo y sobre cómo éste está presente en Vieland, afirmando que, aunque el tiempo de los fantasmas había expirado con la Ilustración, la religión era todavía necesaria para compensar el poder de la ciencia y la razón:

Pero hay otra leccion que darles, y mas importante que esta, atendido el impero actual de nuestras costumbres. Puede decirse que la época de los fanáticos ya ha espirado, y que la supersticion ha perdido tambien su influjo en las vicisitudes y desgracias de la vida humana. Los espectros y las fantasmas apenas conservan nada de su antiguo crédito, y no es de temer que haya muchos Vielands que sacrifiquen

I4 En las citas de Vieland y otros textos del siglo xix se han respetado la acentuación y el deletreo originales. 
á su mujer y á sus hijos engañados por una falsa voz, ó atemorizados por los efectos de la fantasmagoría que ya nadie ignora. Pero es mucho de temer que los espíritus fuertes, y los sabios de la naturaleza, sigan todavía en la ciega persuasion de que bastan los talentos y la sabiduría humana para encontrar y asegurar su felicidad. E1 autor de la novela les hace, pues, ver por el contrario, que el hombre, aunque posea los talentos mas sublimes, y haya adquirido los mas profundos conocimientos en todas las ciencias que él ha formado, si no procura gobernarse por los principios benéficos y filantrópicos que dicta la Religion [sic], no podrá menos de ser tan perjudicial á sus semejantes, como á sí mismo. Tirano de los otros, morirá necesariamente esclavo, y víctima de sus pasiones (I8I8: V-IX).

La parte final de este prólogo está dedicada a las características formales de la traducción, afirmando Monfort que el texto ha sido acortado en relación al «original» francés y adaptado a un público español. La primera característica de esta adaptación es la traducción de los nombres de los personajes y los lugares (Wieland $\rightarrow$ Vieland; Theodore $\rightarrow$ Teodoro; Catherine $\rightarrow$ Catalina; Carwin $\rightarrow$ Carvino; Delaware $\rightarrow$ Delavare, etc.):

Por lo que mira á la traduccion, tengo por muy ridículo encarecer su dificultad, ni prevenir la opinión de los lectores sobre su mérito. Yo solo escribo por ocupar honestamente el tiempo, y ser de alguna manera útil al público, mas no para adquirir nombre de escritor, y mucho menos de traductor, porque sé lo que esto vale en el dia.

Debo por último hacer una advertencia, y es, que en esta traduccion se encontrarán de menos muchas expresiones y cláusulas, y aun capítulos enteros del original. Los gramáticos y traduccionistas mirarán esta licencia como un crímen; pero yo sé que la gente sensata no dejará de conocer los justos motivos que para ello he tenido, y que aplaudirá la rectitud de mi intencion. Pigaul-Maubaillarcq, miembro corresponsal de la Sociedad Filotécnica, dispuso esta novela al gusto y costumbres de su pais, y yo he debido acomodarla á las del nuestro; esto es, el autor frances escribía entre los suyos, y yo traduzco esta obra para los españoles (I8I8: XII-XIV).

La edición de 1826 (impresa en la Imprenta de Gimeno, de Valencia) no incluye este prólogo. ${ }^{15}$ Esta edición se inicia con una nota del editor que resume el prólogo de Luis Monfort (páginas v-vii). Sin embargo, sí es cierto que presenta algunas características que merecen ser tenidas en cuenta. La primera de ellas es que Gimeno diferencia tres tipos de lectores potenciales que podrían sentirse atraídos por Vieland: observadores de las costumbres morales, buscadores de aventuras y lectores cultos que desean comparar Vieland con la versión francesa. La inclusión del segundo grupo es especialmente relevante porque sirve para enlazar esta novela con el género gótico y lo sublime que se había desarrollado en Europa en las décadas precedentes, así como con la estética romántica. Por otro lado, con la inclusión del tercer grupo Gimeno afirma que muchos lectores «[...] los cuales comparando el Vieland español con el Vieland frances han creído ver con sorpresa una novela original, mas bien que una mera version [... ]» (1826: vi). Finalmente, Gimeno también menciona la popularidad de la novela: «Así han desaparecido prontamente todos los ejemplares de la primera edición, y me ha sido preciso acelerar la segunda, que ha

I5 No obstante, una en el primer tomo afirma que los derechos de impresión siguen siendo propiedad de Cabrerizo, habiéndolos adquirido, presumiblemente, en I8I8 con la primera edición. 
revisado el traductor ${ }^{16}$ añadiéndole alguna noticia sobre la electricidad y la ventrilocucion [...]» (I826: VI). Las «notas» que Gimeno menciona merecen ser discutidas de forma independiente.

Según el editor, las notas finales habían sido incluidas por el traductor (y completadas en 1826), y cada una de ellas corresponde a cada uno de los cuatro volúmenes en que se divide Vieland. ${ }^{17}$ La nota correspondiente al primer volumen es un pequeño tratado sobre la electricidad, ofreciéndose este fenómeno como una posible explicación para la misteriosa muerte del viejo Vieland. La nota que va con el segundo volumen debate acerca de la voz humana y la ventriloquía, en relación con la aparición de Carvino. La tercera nota, una versión ampliada de una que aparece en la versión de 1818, hace referencia a una obra titulada Espia en las cortes de los principes cristianos, ó Memorias para servir á la bistoria de este siglo desde 1637 hasta I682. ${ }^{18}$ Más adelante, la nota continúa con una narración sobre Sebastián de Portugal y un tratado sobre la piedra filosofal. Finalmente, la nota que acompaña al cuarto volumen, de nuevo introduce una obra ficticia, en este caso una novela de corte gótico titulada Historia alegórica del principe de Caquemira, ó los prodigios de la ciencia. Con respecto a ella, Monfort destaca una sección titulada «Cartas á Sofía sobre la física, química é historia natural por Mr. Martin». La diferencia radica en que esta sección en concreto sí hace referencia a una obra real (Cartas á Sofía en prosa y verso, sobre la física, quimica é historia natural), escrita por Louis-Aimé Martin (I782-I844) y publicada en Madrid en 1819. La novela ficticia en la que se incluye esta sección narra la historia de un príncipe que abandona sus dominios en busca del fin del mundo y de una mujer infiel. Como conclusión, llega a un palacio subterráneo en el que brazos y piernas se mueven violentamente, $\mathrm{y}$ en el que también hay cabezas parlantes. La descripción que ofrece Luis Monfort recuerda los escenarios del Infierno (ca. I500-1505) de El Bosco (I450-I516), o al Vathek de William Beckford. ${ }^{19}$ La edición de 1839 no incluye ninguna variación sustancial con respecto a la de 1826 .

La notas al pie y finales que aparecen en las sucesivas ediciones también merecen una breve explicación. La edición de I8I8 incluye dos notas al pie bastante ilustrativas en relación a ese sentido de «españolidad» que Monfort buscaba con su traducción. La primera de ellas (p. 8, primer volumen, incluida también en las ediciones de $1826-$ p. 8 - y 1839 - p. 9- $)^{20}$ trata sobre los «camisardos», siendo descritos como «Secta de los hereges reformados ó calvinistas, que ferment en Francia á principios del siglo pasado» (I8I8: 8). ${ }^{2}$ Esta nota es un trasunto del Wieland de Brockden Brown, en el que ya aparece mencionado este grupo protestante: «The book contained an exposition of the doctrine of the sect of Camissards, and an historical account of its origin» (20II: 10). PigaultMaubaillarcq y Luis Monfort decidieron mantener la referencia. Más adelante, en la página 35 ( 34 en la edición de 1826 y 40 en la de 1830 ), hacia el final del capítulo II, hay una nota sobre la muerte del viejo Vieland. En cada una de las ediciones, la nota menciona dos investigadores franceses y dos italianos que, supuestamente, han estudiado el

I6 Esto puede suponer que Monfort seguía vivo en 1826.

I7 La edición de 1818 solo presenta una nota, correspondiente al tercer volumen, como se verá más adelante.

I8 Posiblemente una adaptación del título de L'Espion Turc (1681 [1684]), de Giovanni Paolo Marana (1642-I693).

I9 El conocimiento que, en teoría, tenía Monfort sobre literatura en lengua francesa pudo haberle llevado a haber tenido algún tipo de contacto con esta novela gótica.

20 Presumiblemente, también aparecería en la edición de i8zo.

2I El término «hereges» será sustituido por «herejes» en las ediciones de 1826 y I839. 
fenómeno de la combustión espontánea: ${ }^{22}$ Muraire, ${ }^{23}$ Merille, Maffei ${ }^{24}$ y Fontana. ${ }^{25}$ «El diario de Florencia describe un accidente semejante, y en el de Medicina de Febrero y Mayo de 1783 se hallan otros tan maravillosos, referidos por los señores Muraire y Merille, y las doctas investigaciones de Maffei y de Fontana dan mucha luz sobre esta especie de fenómenos» (1818: 35). La ausencia de ninguna otra mención sobre estos estudiosos lleva a pensar que los nombres fueron simplemente copiados de la versión francesa. Ninguno de ellos aparece en el Wieland de Brown; el capítulo simplemente se cierra con las consabidas preguntas retóricas de Clara, sin más explicación sobre el extraño suceso que acaba de tener lugar:

Was this the penalty of disobedience? this the stroke of a vindictive and invisible hand? Is it a fresh proof that the Divine Ruler interferes in human affairs, meditates and end, selects and commissions his agents, and enforces, by unequivocal sanctions, submission to his will? Or, was it merely the irregular expansion of the fluid that imparts warmth to our heart and our blood caused by the fatigue of the preceding day, or flowing, by stablished laws, from the condition of his thought? (2OII: I8).

La edición de 1826, hacia el final del capítulo viI del cuarto volumen (p. 139), incluye una nota en la que se menciona a un tal Rainaldo, supuestamente un filósofo especialista en temas americanos y del que nada se sabe. Entre las adiciones hechas por antiguos propietarios, por ejemplo, en la página 7 del primer volumen se incluye una nota en la que las palabras «las mientes» se han tachado y sustituido por «La imaginacion». ${ }^{26}$

La edición de I839 incluye la particularidad de contar con una lista de ciudades en las que Cabrerizo había distribuido Vieland. Aparte de las capitales de provincia que cabría esperar, tres localizaciones llaman poderosamente la atención: Gibraltar, La Habana y Puerto Rico. Esto significa que la traducción de Luis Monfort había sido dada a conocer en el extranjero en i839 y que Wieland, paradójicamente, había regresado a América vía Francia y España, sin una sola mención a su autor americano original. ${ }^{27}$

22 Para más información sobre este fenómeno y su relación con la obra, véase, por ejemplo, la noticia anónima titulada «Letter respecting and Italian priest, killed by an electric commotion, the cause of which resided in his own body», publicada en The American Museum, or, Universal Magazine de Filadelfia en abril de I792 (incluida en la edición de Norton de Wieland referenciada en la Bibliografía o la información aportada por los editores de Charles Brockden Brown's Wieland, Ormond, Arthur Mervyn, y Edgar Huntly (también referenciada en la Bibliografía).

${ }_{23}$ Posiblemente Honoré Muraire (I75O-I837), un político francés que dedicó gran parte de su vida a promocionar la masonería. Junto con Merille, entre 1779 y I782, ofreció algunos ejemplos y evidencias sobre la combustión espontánea en el Journal de Médecine de París.

24 Posiblemente Francesco Scipione, marqués de Maffei (I675-I755), un escritor y crítico de arte italiano. No obstante, también realizó publicaciones en el campo de la Filosofía. Su primer trabajo en este campo fue la Istoria teologica delle doctrine e delle opinione corse ne cinque primo secoli della chiesa in proposito della divina grazia, del libero arbitrio e della predestinazione, contra los jansenistas (I742). Sin embargo, su obra más interesante para lo que nos ocupa fue su Arte magica annichilata (I754), en la que trata de lo sobrenatural, la magia y las brujas. Este panfleto sería incluido posteriormente en el Traité sur les apparitions des esprits et sur les vampires ou les revenans de Hongrie, de Moravie, Eंc. (I75I) de Antoine Augustin Calmet (I672-I757).

25 No se ha encontrado información acerca de Fontana.

26 En el mismo volumen, en la página 77, una nota manuscrita reza «Leed y hallareis cosas prodigiosas mas.» (una reescritura de la frase que aparece en la portada). A título anecdótico, parece que este propietario se hubiese obsesionado con Vieland por todas las notas manuscritas que incluyó: en la página II8, la palabra «conversación» se cambió a «conservación» en una nota al pie, en la que también viene escrito «Moya lo enmendó». Al final del primer volumen, en una de las páginas de cortesía, también añadió «Nada hay de fabuloso» y, en la página I2I (segundo volumen), sobre el encabezado del Capítulo VII, escribió «Lee y te admirarás».

27 Esta edición cuenta con una única nota manuscrita, que aparece hacia el final del prólogo y en la que se menciona a una antigua propietaria: «Propiedad de María». 
Finalmente, de entre todas estas notas, una merece una especial atención. Esta aparece al final del cuarto volumen en todas las ediciones y lleva por título «Nota». Hace referencia a la vida de Clara tras los trágicos eventos de los que es testigo, así como a su fallecimiento:

En efecto murió la desventurada Clara al terminar estas memorias que escribió á instancias de un pariente lejano, que vivia en Sajonia, de donde se ha visto descendia su familia. Todos los que figuran en esta desastrosa historia perecieron desgraciadamente. ¡Lastimoso cuadro de las funestas consecuencias de una educacion fanática y supersticiosa como la de Vieland, privada de la ilustracion del Evangelio; y trágica perspectiva de las calamidades, víctimas y ruinas que puede causar un hómbre, al cual, como al ominoso Carvino, no enfrene la religion cristiana su altivo saber y fogosas pasiones! (I8I8: 223).

Esta «Nota» se utiliza como un recurso para acabar la historia de forma abrupta tras la batalla en el escondite de Carvino. Como el lector sabrá, en el Wieland de Brockden Brown, Clara es la narradora hasta el final:

I leave you to moralize on this tale. That virtue should become the victim of treachery is, no doubt, a mournful consideration; but it will not scape your notice, that the evils of which Carwin and Maxwell were the authors, owed their existence to the errors of the sufferers. All efforts would have been ineffectual to subvert the happiness or shorten the existence of the Stuarts, if their own frailty had not seconded these efforts. If the lady had crushed her disastrous passions in the bud, and driven the seducer from her presence, when the tendency of his artifices was seen; if Stuart had not admitted the spirit of absurd revenge, we should not have had to deplore this catastrophe. If Wieland had framed juster notions of moral duty, and of the divine attributes; or if I had been gifted with ordinary equanimity of foresight, the double-tongued deceiver would have been baffled and repelled (20II: I8I).

En relación a la traducción, este estudio tan solo se centrará en aquellos aspectos o pasajes particulares modificados por Luis Monfort. Como se ha dicho más arriba, la descripción que se ofrecerá hará referencia, fundamentalmente, a los dos primeros volúmenes, en los que se sigue el argumento ideado por Charles Brockden Brown. Siguiendo la narración de Clara, la mayor parte de estas singularidades están relacionadas con el concepto de «religión verdadera» la carencia que de ella tienen los personajes de la novela. Por ejemplo, hacia la mitad del primer capítulo (edición de i818), aparece una reflexión de Clara acerca de este concepto como un elemento que habría llevado a su padre a fracasar en su misión evangelizadora en América: «[...] las falsas ideas que habia ido adquiriendo. Entusiasmo, vocacion, por cierto sublimes, si fueran inspiradas por el santo celo de la verdadera creencia» (I8I8: I2). Este párrafo no aparece en la versión francesa, en la que tan solo se habla de la determinación del viejo Wieland de hacerse misionero:

Il fut d'abord effrayé des dangers et des fatigues auxquelles la vie d'un missionaire allait l'exposer, et cette appréhension le rendit fertile en expédiens pour retarder l'exécution de ce projet, sans qu'il lui fût sependant possible de cesser de se eroire destine à remplir ce pénible devoir (Pigault-Maubaillarcq, I808: 20). 
Brown tampoco incluye la mencionada referencia, sino que finaliza el pasaje como sigue:

He had imbibed an opinion that it was his duty to disseminate the truths of the gospel among the unbelieving nations. He was terrified at first by the perils and hardships to which the life of a missionary is exposed. This cowardice made him diligent in the invention of objections and excuses; but he found it impossible wholly to shake off the belief that such was the injunction of his duty. The belief, after every new conflict with his passions, acquired new strength; and, at length, he formed a resolution of complying with what he deemed the will of heaven (20Ir: II).

Otra escena adaptada por Monfort es en la que transcurre la muerte del viejo Vieland a causa de una combustión espontánea (capítulo II). Aquí, Monfort da menos información que Pigault-Maubaillarcq. Mientras que la versión francesa se extiende tres párrafos narrando el hecho, la traducción de Monfort lo despacha en unas pocas líneas:

Se supo por sus incoherentes respuestas, que mientras se hallaba en silencio ocupado en la oracion con el terror é inquietud, que se apoderaban de él en aquel acto, se lanzó de improviso un ligero resplandor al traves del edificio, que su imaginacion le presentó como dimanado de alguno que se le presentaba por las espaldas. Iba á volver la cabeza para cerciorarse, cuando se sintió acometido de una violenta conmocion por todos sus miembros, al mismo instante que un fuego voraz, prendiendo en sus vestidos los redujo á cenizas hasta abrasarle su cuerpo (I818: 32).

Paradójicamente, mediante el resumen que hace Monfort, esta versión está más próxima al original estadounidense que la francesa:

By this imperfect account, it appeared, that while engaged in silent orisons, with thoughts full of confusion and anxiety, a faint gleam suddenly shot athwart the apartment. His fancy immediately pictured to itself, a person bearing a lamp. It seemed to come from behind. He was in the act of turning to examine the visitant, when his right arm received a blow from a heavy club. At the same instant, a very bright spark was seen to light upon his clothes. In a moment, the whole was reduced to ashes. This was the sum of the information which he chose to give. There was somewhat in his manners that indicated an imperfect tale (Brown, 20II: 17).

El capítulo III retoma el tema religioso, y en él Monfort introduce algunas imprecaciones con el objetivo de resaltar las virtudes del Catolicismo frente al Protestantismo. A diferencia de lo que ocurre en la versión francesa, en Vieland se afirma que la religión de la que carecen Clara y Teodoro es una doctrina revelada, procedente directamente de Dios: «Nuestra educacion por desgracia no habia sido bajo el sagrado estandarte de una religion revelada» (I8I8: 40); por el contrario, la versión de Pigault-Maubaillarcq no hace mención a este sentido de «revelación», sino que su traducción solo dice que la educación que los Wielands habían recibido había estado totalmente carente de adoctrinamiento religioso: «Notre éducation n'avaitété dirigée sous l'étendart d'aucune religion particulière» (I808:49). Poco después de la mencionada imprecación, Monfort continúa desarrollando su teoría, dando a Clara la oportunidad de expresar su desesperación a la hora de afrontar las tribulaciones que se le presentan sin contar con el consuelo de la religión verdadera: «iLa religion! ¡Ah! ¡cuan preciosa nos hubiese sido en la adversidad, y el infortunio!» (I818: 4I). 
Pigault-Maubaillarcq incluye su propia versión de estas tribulaciones, pero sin el énfasis que Monfort da a la influencia de la religión en la vida: «La religion est, sans doute, bien précieuse, sour-tour quand on la considère comme una consolation dans l'infortune [...]» (1808: 49-50). El Wieland de Brown coincide con la versión de Pigault-Maubaillarcq, destacando la educación natural, liberal e ilustrada que Theodore y Clara habían recibido: «Our education had been modelled by no religious standard. We were left to the guidance of our own understanding, and the casual impressions which society might make upon us. My friend's temper, as well as my own, exempted us from much anxiety on this account» (20Ir: 20).

Otro pasaje interesante (capítulo viI) es que narra la estancia de Carwin en España (en Murviedro - Sagunto-) y cómo Pleyel recuerda el momento en que allí se encontraron. Tanto Pigault-Maubaillarcq como Luis Monfort toman este pasaje de la versión de Brockden Brown sin ningún cambio:

In answer to my inquiries, he informed me that, three years before, he was a traveller in Spain. He had made an excursion from Valencia to Murviedro with a view to inspect the remains of Roman magnificence, scattered in the environs of that town. While traversing the scite of the theatre of old Saguntum he lighted upon this man, seated on a stone, and deeply engaged in perusing the work of the deacon Marti. A short conversation ensued, which proved the stranger to be English. They returned to Valencia together (20Ir: 54).

Sin embargo, este hecho es extremadamente paradójico porque, si se presta atención al tercer volumen de Vieland, Monfort sigue la nueva línea argumental de PigaultMaubaillarcq acerca de Carwin y sus orígenes, sin hacerse mención a su visita a España (o a sus años en Irlanda, por ejemplo). ${ }^{28}$

El volumen II también incluye una línea argumental novedosa en algunos de sus capítulos. Por ejemplo, el capítulo vir narra una historia de amor de Carwin antes de llegar a casa de los Vielands: bajo nombre falso seduce a la hija de su casera. Sin embargo, lo verdaderamente interesante sobre este pasaje está mucho más desarrollado en la versión de Monfort que en la de Pigault-Maubaillarcq. El traductor español incluye detalles que no aparecen en la versión francesa, como los libros en árabe, portugués y español que se descubren entre las posesiones de Carvino: «[...] no hallamos mas efectos ni papeles que unos manuscritos, y algunos libros en lengua portuguesa, española y árabe» (I818: 158).

En la versión española, este volumen también añade argumentos a la discusión de la religión verdadera frente a la falsa. Después de que Teodoro es encarcelado por sus crímenes, Clara y un tío que no se ha mencionado anteriormente se ponen a investigar la procedencia e identidad de Carvino. Entre los testimonios que escuchan durante el proceso, uno de ellos (oído en Filadelfia) es especialmente relevante. En él, un testigo menciona que conoce a un ministro presbiteriano llamado Broun, y el Presbiterianismo es inmediatamente clasificado como una secta: «Respondiome que se llamaba Broun; que era ministro del culto presbiteriano, y que estaba encargado de la direccion espiritual de las de aquella secta [...]» (1818: 174).

Como se ha podido ver, los cambios y adaptaciones de Luis Monfort, aunque no excesivamente abundantes con respecto a la versión de Pigault-Maubaillarcq, contribuyen a construir ese «sentido nacional» que tanto el traductor como los editores habían anunciado,

28 Aunque en este volumen se cuenten, de forma extensiva, los viajes de Carwin por distintos países, como Egipto (donde aprende el arte de la ventriloquía), Italia, Portugal, Austria, etc. 
como se ha expuesto más arriba. Sobre todo, la inclusión del debate religioso pudo haber resultado de utilidad a la hora de atraer lectores burgueses y de corte conservador. También pudo haber tenido el propósito de evitar los problemas que María José Alonso Seoane menciona en su artículo, problemas que habrían venido de la Inquisición como institución censora de la cultura.

La historia que aparece en el tercer volumen no se va a analizar en profundidad debido a que, como se ha mencionado, no guarda relación con el Wieland de Brown. Sin embargo, sí que merece alguna atención debido a las circunstancias editoriales que rodearon su publicación. Siguiendo a Miriam López Santos y José F. Montesinos, es posible encontrar cierta información acerca de cómo este volumen vio la luz. Debido a la historia de aventuras que narra, los editores (especialmente Mariano de Cabrerizo) decidieron venderlo de forma independiente bajo el título Carvino ó el hombre prodigioso. Como se ha visto en relación a Vieland, el nombre de Pigault-Maubaillarcq no aparece mencionado ni una sola vez. Montesinos menciona dos ediciones para este spin-off de la novela, una publicada en 1830 (presumiblemente junto con la tercera edición de Vieland) y otra en I84I. Montesinos, desconociendo la versión de Pigault-Maubaillarcq, afirma: «[...] no sé hasta qué punto original o traducida» (1982: 262). Si se compara esta publicación con el tercer volumen de cualquiera de las ediciones del Vieland de Monfort se apreciará que son idénticas. Por otro lado, López Santos, ofrece algo más de información. Utilizando el libro de Montesinos como base, afirma:

Montesinos (I98I: 262) identifica, asimismo, la novela Carvino o el hombre prodigioso como perteneciente a Pigault-Maubaillarcq en I830 y otra vez en I84I, considerando que puede tratarse de una continuación de La Familia de Vieland. Una lectura de la misma me permite descubrir uno de sus personajes y el mismo tema de la ventrilocución. Sin embargo, se trata de una narración de lances fabulosos y extraordinarios en los que está ausente toda experiencia de terror. En cuanto si pertenece al mismo autor, ${ }^{29}$ no puedo asegurarlo firmemente aunque no parece coincidir con los títulos de sus novelas originales (2010: 47, n. 8I).

Como se ha visto para las distintas ediciones de Vieland, Carvino no se publicó de forma totalmente aislada, sino que se acompañó de algunos otros textos que merecen ser analizados independientemente $3^{\circ} \mathrm{Al}$ comienzo de Carvino aparece un prólogo titulado «Vista preliminar», no habiéndose podido determinar si fue una adición de Monfort o de Cabrerizo, o una traducción de otro texto, pues aparece sin firma. Entre los detalles que se incluyen en esta «Vista», de nuevo se insiste en el sentido moral que tanto Pigault-Maubaillarcq como Luis Monfort querían darle a sus traducciones. Por ejemplo, en él se dicen cosas como: «Su primer delito fue la ingratitud, y los Padres Jesuitas su primera víctima» (I830: VII) o «La educacion física precedió en Carvino á la educacion moral [...]» (I830 [I84I]: XVIII). Más allá de estas disquisiciones morales, quienquiera que fuese el autor dedica nueve páginas a referenciar una obra ya discutida: Espía en las cortes de los Principes cristianos, ó memorias para servir á la historia de este siglo desde I637 hasta I682. Como se ha dicho más arriba, aunque este escrito se presenta como una obra real, su existencia no ha podido ser probada. ${ }^{3 \mathrm{I}}$ Esta

3I No obstante, existe la posibilidad de que los fragmentos concernientes a Sebastián de Portugal tengan un origen en una obra real. Entre 1775 y 1796, el editor barcelonés Carlos Gibért y Tutó publicó, por primera vez, una comedia inédita de Francisco de Quevedo (I580-1645) titulada El Rey D. Sebastian y portugues mas heroico: Comedia Heróica en tres actos, Barcelona, Imprenta de Carlos Gibért y Tutó. 
«Vista preliminar», sin embargo, va un paso más lejos, reproduciendo una cita de nueve páginas supuestamente extraída de esta obra ficticia. Esta cita es una narración de cómo Sebastián de Portugal murió en la Batalla de Alcazarquivir en 1578 , y cómo su leyenda todavía perduraba en Europa. ${ }^{32}$ Después de la larga cita, la «Vista» continua resumiendo la vida de Carvino (y, consecuentemente, la novela en sí misma), un cometido que ya se había iniciado antes de la cita. Sin embargo, el fragmento más importante de la «Vista preliminar» aparece justo antes de la referencia a Espia en las cortes de los Príncipes cristianos, ó memorias para servir á la historia de este siglo desde I637 hasta I682. El párrafo previo incluye la frase «[...] pero sus funestas pasiones, despues de haber cubierto de muertes y de luto á la infeliz familia de Vieland» (I830 [I84I]: VII-VIII). Estas líneas ofrecen una posible solución al mayor misterio de Wieland desde Brown a Monfort: ¿de dónde procedían las voces escuchadas por Theodore/Teodoro?

Una vez que Carvino concluye se incluyen otras dos notas. La primera de ellas (titulada «Nota»), como ocurría con la «Vista preliminar», aparece sin firma. Esta sigue un modelo similar a la nota incluida al final del cuarto volumen de Vieland y sirve para cortar abruptamente la historia. En este caso, la «Nota» sirve para resumir el cuarto volumen de Vieland que, aunque no se incluye en Carvino, cierra la historia del infausto personaje y la rebelión indígena que intenta organizar:

El éxito de esta temeraria empresa, con cuyo feliz resultado hubiera sido Carvino un monarca poderoso, fue enteramente contrario á sus esperanzas: pereció en ella este hombre extraordinario, y con él todos los gefes Esquimales, Abenaquíes, Chancotinis, y otras tribus que pueblan las riberas del rio S. Lorenzo; y Clara Vieland, libertada milagrosamente de esta catástrofe, vino á Marsella despues de mil contratiempos á exhalar el último suspiro, segun se hace relacion en el tomo cuarto y último de LA FAMILIA DE VIELAND (I818: 295).

La segunda de estas notas, titulada «Nota del traductor», de nuevo hace referencia a Espía en las cortes de los Príncipes cristianos, ó memorias para servir á la historia de este siglo desde I637 hasta I682. En este caso, como se ha visto más arriba cuando se analizaban los dos primeros volúmenes de Vieland, el fragmento incluido en Carvino trata sobre la piedra filosofal y acerca de cómo Carvino aprendió sus misterios (junto con el arte de la ventriloquía) durante su estancia entre los ermitaños de Egipto.

\section{El COMPENDIO}

Aparte de la importancia de Vieland y sus características formales, su relevancia se puede cifrar en relación a una nueva obra literaria que se produjo bajo su influencia directa. La novela alcanzó tal popularidad que uno de sus lectores compuso un tratado sobre su propia familia ofreciendo un resumen de Vieland como introducción a sus propias desgracias. En I845, Pablo Galende García (del que nada se sabe) publicó una obrita titulada Compendio que demuestra la verdadera esencia de la historia de las desgraciadas familias de Vieland, y D. Francisco II, rey de Narea, apellidado Carbino. Esta obra constituye un intento de ganar popularidad de cara a la resolución de un pleito en el que estaba inmersa su familia. Sin embargo, la primera parte de este tratado es el mencionado resumen y, a través de él, el autor trata asimilar sus propias desgracias a las de los Vielands. Este

32 Como el lector sabrá, el movimiento romántico recuperaría la figura de este rey como modelo de valentía y virtudes caballerescas. 
resumen presenta la historia como un hecho real que ocurrió en el pasado; Clara, en consecuencia, no sería una narradora, sino una cronista.

Como se ha visto en la sección anterior, la versión española de Wieland (hasta cierto punto, también la francesa) intenta moralizar la novela, añadiendo o enmascarando pasajes o frases que aparecen en la versión original de Brown. Por ejemplo, Pablo Galende García debate acerca de la religión de Teodoro y Clara, situándola en el centro de la tragedia:

Su educación por desgracia no había sido dirigida bajo el sagrado estandarte de una religión revelada: no conocían mas principios religiosos que el reconocimiento que les inspiraba su misma felicidad, y el magestuoso espectáculo de la naturaleza: su culto solo consistia en sentimientos vagos y superficiales que no estaban sujetos á ninguna práctica esterior (1845: 12 ).

El autor incluso trata de solventar algunos de los pasajes más oscuros de la traducción de Monfort, dando posibles explicaciones a eventos que el lector no puede conocer sólo con la información que se da en la novela, como por ejemplo el intento de Teodoro de asesinar a Clara. Para ello, Galende García procede como un crítico literario, dando evidencias para persuadir al lector:

Si se fija la atencion en el estado en que se presentó Teodoro á su hermana Clara para asesinarla por última vez,... ensangrentado,... y en que no se volvió a hablar de Agueda, letra $\mathrm{H},{ }^{33} \ldots$ vendremos á presumir con fundamento que sin duda la asesinó dicho Teodoro, antes de presentarse á su dicha hermana!... Tom. $44^{\circ}$, fol. nIo y siguientes. ¡Y qué diremos de la salida de los calabozos!... ¡ ¿Quién se la proporcionaria!... ¡Tendria o buscaría algun medio ó influjo Pleyel y la baronesa, su esposa, para que lo consiguiese!... (I845: I9).

Tras este aviso (titulado «Advertencia») el autor continúa exponiendo el pleito en el que familia estaba envuelta.

Como cabe esperar, este tratado sólo se editó una vez. La Biblioteca Nacional alberga una copia, la cual incluye un árbol genealógica de los Vielands, los Pleyels y Carvino (véase la Figura 5). 


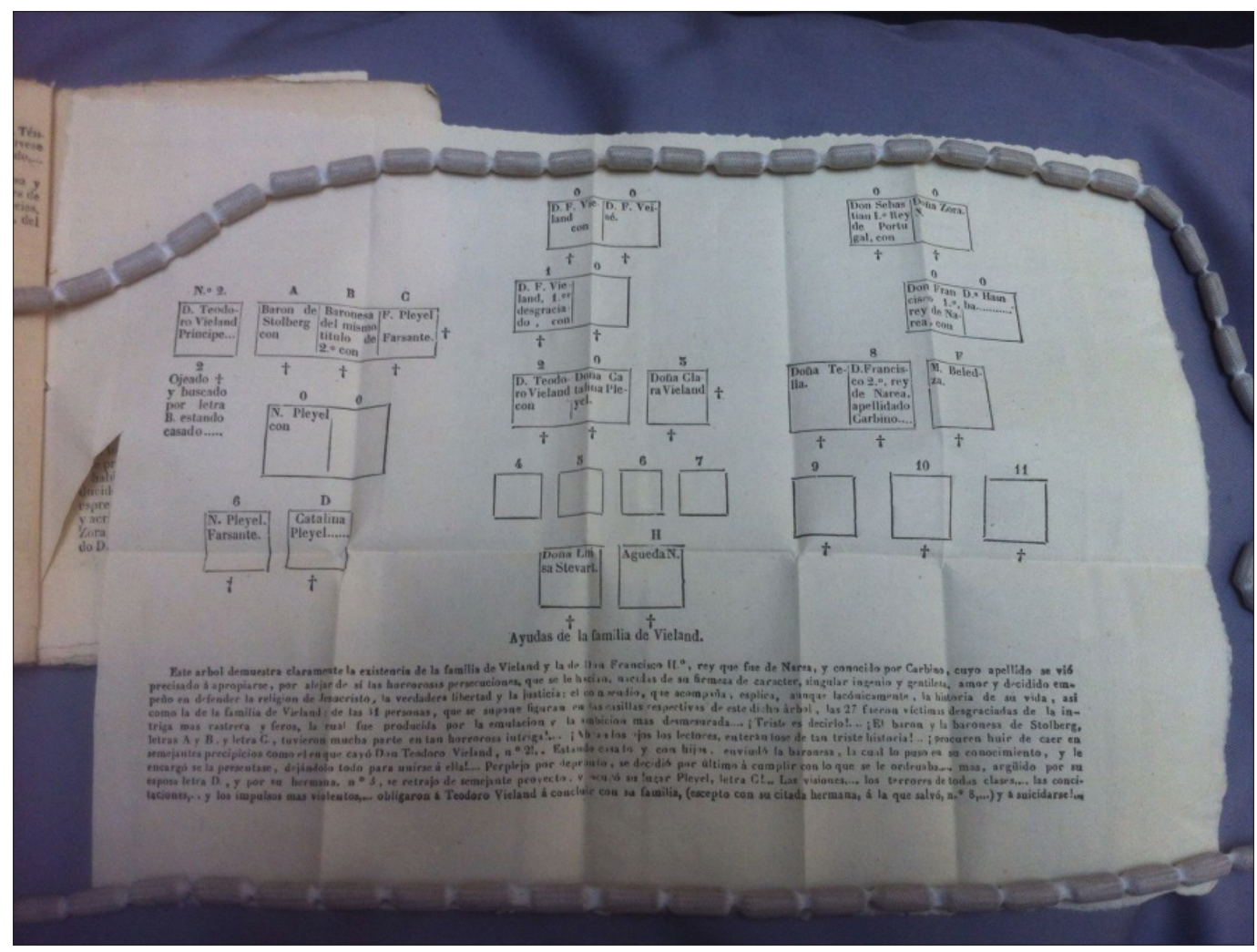

Figura 5: Árbol genealógico de los Vielands, los Pleyels y Carvino.

Fuente: fotografía del autor con permiso de la Biblioteca Nacional

Esta copia es también interesante porque contiene algunas notas marginales en las que se exponen las opiniones de un antiguo propietario. Este lector llegó a la conclusión de que fueron los perversos actos de los Pleyels (especialmente Catalina) las que hicieron enloquecer a Teodoro y lo llevaron a asesinar a su familia.

La existencia de la obra de Pablo Galende García, más allá de su poca calidad literaria, es útil a la hora de probar la relevancia que Vieland tuvo en la España de la primera mitad de siglo xIX, con un volumen de lectores tan amplio que incluso se produjo una publicación paralela, de corte crítico, al respecto. Sin embargo, una vez más debe señalarse el hecho de la falta de conocimiento de qué obra se estaba leyendo en realidad, pues esto podría haber cambiado su recepción y cómo ha llegado hasta nosotros.

\section{El CONTEXTO SOCIOCULTURAL}

Como se ha dicho más arriba, entender el contexto en el que Vieland se publicó es crucial para dictaminar su relevancia. Como también se ha dicho en párrafos anteriores en relación a la versión francesa de Pigault-Maubaillarcq, el clima de libertad cultural de la Francia napoleónica era suficientemente sólido como para permitir el florecimiento de la novela gótica. Así, es fácilmente comprensible que el Wieland de Charles Brockden Brown se publicase en Calais en I808. Sin embargo, en España las circunstancias eran diametralmente opuestas tras la Guerra de Independencia y la restauración de la Monarquía 
Absoluta con Fernando VII. ${ }^{34}$ Estas últimas décadas de Absolutismo coincidieron con las últimas de existencia de la Inquisición, abolida en 1834. Por lo tanto, las tres primeras ediciones de Vieland se publicaron bajo el control del Santo Tribunal sobre la cultura. Henry Kamen, entre otros, afirma al respecto:

In its early years the attempts of the Inquisition to identify and control erroneous beliefs were directed mainly against ideas, practices and words, rather than against writings. In pre-modern Europe culture and belief were primarily expressed orally; only occasionally did the inquisitors find it necessary to attack the objects through which ideas could be transmitted, namely, books.

Since medieval times, books were produced in manuscript form and copied by hand where necessary; they were rare, expensive and hard to find, but in a Europe where illiteracy was the order of the day the demand was small. Still, the inquisitors by no means neglected their significance. When Hebrew books and the Talmud were found in the possession of conversos, they were seized and destroyed. The inquisitors also seem from an early period to have frowned on books about magic and astrology (2014: 950-952).

El anticlericalismo del que muchas novelas góticas hacían gala (con ejemplos como The Monk [1796], The Italian [1797], Melmoth the Wanderer [1820], etc.), había hecho de ellas un objetivo fácil para la censura eclesiástica en diversos países de Europa, entre los que se contaba España. Por ello, las novelas góticas (que ya habían sido calificadas de «literatura terrorista» ${ }^{35}$ fueron incluidas en el grupo de los libros heréticos conforme el género cobró la suficiente fama como para ser conocido a nivel continental. Miriam López Santos también hace mención a la censura gubernamental sobre la novela gótica:

El mantenimiento de aquellas viejas supercherías se vio reforzado gracias al cultivo de la nueva literatura de vertiente irracional que llegaba desde Inglaterra. La situación adquirió tal envergadura, ante los escasos o nulos resultados que obtuvieron las recomendaciones de los iluministas, por una parte, y los recientes «peligros» que acechaban a nuestro país, por otra, que el gobierno se vio forzado a actuar de manera implacable a través de la censura [...] (2010: 24).

Aunque la influencia de la Inquisición no fuese tan fuerte como en los tiempos de Felipe II o Felipe IV, su influjo aún tenía presencia en diferentes aspectos de la vida diaria de los ciudadanos. Al respecto, Helen Rawlings afirma que, cuando la institución fue restablecida por Fernando VII «Its purpose was to guard against the progressive influences introduced into Spain during the period of French occupation and restore respect for the faith» (2006: I43). Dado que las persecuciones contra los judíos y los herejes habían finalizado en el siglo xviII, el nuevo interés de la Inquisición se centraba en el control sobre la cultura, como Rawlings continúa afirmando: «The revived Inquisition justified its activities as censor on the grounds that it was necessary "to root out as soon as possible every kind of publication in any way contrary to the doctrine of holy religion and to the fidelity owed to the Sovereign" (Callahan, I984, p. II3)» (2006: I43). En este ambiente, los problemas que María José Alonso Seoane menciona con respecto a Cabrerizo no

34 Quizá con el breve paréntesis liberal de $1820-1823$.

35 Véanse la mencionada referencia a Les Crimes de l'Amour, del marqués de Sade, u obras como «The Blasphemy of The Monk» (1797), de Samuel Taylor Coleridge (1772-1834), por ejemplo. Más recientemente, la crítico Angela Wright (2007) ha vuelto sobre esta calificación en su libro Gothic Fiction, Basingstoke, Palgrave. 
debieron ser una excepción. Más excepcional es el hecho de que Vieland se publicase tres veces durante esos años, dando lugar incluso a dos obras paralelas (Carvino y el Compendio). Con los «antecedentes» de esta novela (tratando temas religiosos y "producida» en Francia) resulta sorprendente que Estévan se decidiese a publicarla por primera vez en I8I8, incluso con las adiciones morales de Pigault-Maubaillarcq y Luis Monfort. Como se ha mencionado más arriba, Monfort, en su tarea de traductor, nunca fue consciente de la labor que estaba llevando a cabo: introducir la literatura estadounidense en España y traducir una novela gótica que «puentearía» el control inquisitorial.

\section{Conclusiones}

Como se ha visto en las páginas precedentes, Wieland no era desconocido para el público español antes de 1992. Mediante el análisis de las cuatro ediciones que utilizan la traducción de Luis Monfort, ha sido posible probar el interés de los lectores tanto por la novela gótica como por obras concernientes a temas americanos. Aun teniendo en cuenta la faceta moral de que se intentó imbuir a la novela, todos los textos menores analizados dan cuenta de la importancia que Vieland tuvo durante la primera mitad del siglo xix. El Compendio y las notas demuestran que Vieland no fue una novela oscura, sino que gozó de cierta aclamación tanto por parte de la crítica como del mercado. Por otro lado, esta novela marca el comienzo de un cierto interés por los Estados Unidos, un país nuevo y prácticamente desconocido para la mayoría de los españoles en una fecha tan temprana como 1818. La publicación de Vieland también supuso la introducción de la novela gótica en el panorama cultural de la España de la Inquisición. Con este artículo se ha querido ofrecer luz sobre la relevancia de la labor de Luis Monfort, y recuperar del olvido de la historia la primera versión española del Wieland de Brockden Brown (presumiblemente la segunda en Europa). La edición de Agustín Izquierdo contaba, pues, con algunas carencias que el presente estudio ha contribuido a subsanar: existía una traducción anterior, con una importante labor editorial en torno suyo, como ahora ha quedado expuesto para el lector y el crítico.

\section{BibLiOgRAFÍA}

(I830 [1841]), Carvino ó el hombre prodigioso [trad. Dr. D. Luis Monfort], Valencia, Imprenta de Cabrerizo.

(1842), Catálogo de los libros de fondo y surtido de la Imprenta y Libería de Cabrerizo, formado para los SS. Libreros de América, Valencia [del Cid], Imprenta de D. Mariano de Cabrerizo.

(1818), La familia de Vieland, ó los prodigios [trad. Dr. D. Luis Monfort], Valencia, Imprenta de Estévan.

(I826), La familia de Vieland, ó los prodigios [trad. Dr. D. Luis Monfort], Valencia, Imprenta de Gimeno.

(I830), La familia de Vieland, ó los prodigios [trad. Dr. D. Luis Monfort], Valencia, Imprenta de Cabrerizo.

(1839), La familia de Vieland, ó los prodigios [tran. Dr. D. Luis Monfort], Valencia, Imprenta de Cabrerizo.

(20II), «Letter respecting an Italian priest, killed by an electric commotion, the cause of which resided in his own body», en Wieland and Memoirs of Carwin the Biloquist, Nueva York, Norton. 
Alonso Seonne, María José (2002), «El debate sobre el Romanticismo y su temprana defensa en la traducción de Corinne, de Mme. De Staël, por Juan Ángel Caamaño», en Romanticismo 8: Actas del VIII Congreso (Saluzzo, 2I-23 de marzo de 2002). Los románticos teorizan sobre sí mismos, Bolonia, Il Capitello del Sole.

Benezit, Emmanuel (1999), Dictionnaire des peintres, sculpteurs, dessinateurs et graveurs, Paris, Gründ.

Brown, Charles Brockden (2009), Charles Brockden Brown's Wieland, Ormond, Arthur Mervyn, y Edgar Huntly. Indianápolis, Hackett Publishing Company, Inc.

- (20II), Wieland and Memoirs of Carwin the Biloquist, Nueva York, Norton. (1992), Wieland o la transformación, Madrid, Valdemar.

Cabrerizo, Mariano de (I854), Memorias de mis vicisitudes políticas desde I820 á I836, Valencia, Imprenta de don Mariano de Cabrerizo.

Cavero Hernández, María Pilar (2013), Bibliografía de la historia de la imprenta valenciana, Trabajo Fin de Grado (Universidad Politécnica de Valencia).

Charras, Françoise (I999), «Variations et anamorphoses sur le mode gothique, les traductions en français de Wieland au XIX ${ }^{\mathrm{e}}$ siécle», Profils Americains, pp. I9I-2I2.

Davidson, Cathy N. (2004), Revolution and the Word. The Rise of the Novel in America, Oxford, Oxford University Press.

Esplin, Emron y Margarida Vale de Gato (eds.) (20I4), Translated Poe, Bethlehem, PA, Lehigh University Press.

Galende García, Pablo (I845), Compendio que demuestra la verdadera esencia de la historia de las desgraciadas familias de Vieland, y D. Francisco II, rey de Narea, apellidado Carbino, Madrid, Imprenta de D. B. A. de Sopetran.

Izquierdo, Agustín (1992), «Introducción», en Charles Brockden Brown, Wieland o la transformación, Madrid, Valdemar, pp. 3-10.

Kamen, Henry (2014), The Spanish Inquisition. A Historical Revision [en línea], New Haven, CT, Yale University Press [Fecha de consulta: 28/12/2018], disponible en https://bit.ly/2LgA8h2.

López Santos, Miriam (2010), La novela gótica en España (I788-I833), Vigo, Editorial Academia del Hispanismo.

Marana, Giovanni Paolo (I742 [r68I]), L'Espion Turc dans les Cours des Princes Chrétiens, ou Lettres de Memoires d'un Envoyé secret de la Port dans les Cours de l'Europe; ou l'on voit Les Découvertes qu'il a faites dans toutes les Cours où il s'est trouvé, avec des Dissertations curieuses sur leurs Forces, leur Politique E̊ leur Religion, Londres, Dépens de la Compagnie.

Martin, Louis-Aimé (I8I9), Cartas á Sofía en prosa y verso, sobre la física, química é historia natural, Madrid, Imprenta Real.

Montesinos, José F. (I982), Introducción a una bistoria de la novela en España en el siglo XIX. Seguido del esbozo de una bibliografía española de traducciones de novelas 1800-1850, Madrid, Castalia.

Pigault-Maubaillarce (I808), La famille Wieland, ou les prodiges, traduction libre d'un manuscrit américain, Calais, Moreaux.

Quevedo, Francisco de (ca. I775-I796), El Rey D. Sebastian y portugues mas heroico: Comedia Heróica en tres actos, Barcelona, Imprenta de Carlos Gibért y Tutó.

Rawlings, Helen (2006), The Spanish Inquisition, Oxford, Blackwell.

Rigal Aragón, Margarita (20I4), «A Historical Approach to the Translation of Poe's Narrative Works in Spain», en Emron Esplin y Margarida Vale de Gato (eds.), Translated Poe, Bethlehem, PA, Lehigh University Press, pp. 13-23.

SADE, Donatien Alphonse François de (I990), «The Fruit of Revolutionary Tremors», en Victor Sage (ed.), The Gothick Novel. A Casebook, Londres, Macmillan, pp. 48-49. 